\title{
Microsaccades Are Coupled to Heartbeat
}

\author{
Sven Ohl, ${ }^{1,2}$ Christian Wohltat, ${ }^{3}$-Reinhold Kliegl, ${ }^{3}{ }^{-0}$ Olga Pollatos, ${ }^{4}$ and $\odot$ Ralf Engbert ${ }^{3}$ \\ ${ }^{1}$ Bernstein Center for Computational Neuroscience Berlin, 10115 Berlin, Germany, ${ }^{2}$ Department of Psychology, Humboldt Universität zu Berlin, 12489 \\ Berlin, Germany, ${ }^{3}$ Department of Psychology, Universität Potsdam, 14476 Potsdam, Germany, and ${ }^{4}$ Institute of Psychology and Education, Universität \\ Ulm, 89069 Ulm, Germany
}

During visual fixation, the eye generates microsaccades and slower components of fixational eye movements that are part of the visual processing strategy in humans. Here, we show that ongoing heartbeat is coupled to temporal rate variations in the generation of microsaccades. Using coregistration of eye recording and ECG in humans, we tested the hypothesis that microsaccade onsets are coupled to the relative phase of the R-R intervals in heartbeats. We observed significantly more microsaccades during the early phase after the $\mathrm{R}$ peak in the ECG. This form of coupling between heartbeat and eye movements was substantiated by the additional finding of a coupling between heart phase and motion activity in slow fixational eye movements; i.e., retinal image slip caused by physiological drift. Our findings therefore demonstrate a coupling of the oculomotor system and ongoing heartbeat, which provides further evidence for bodily influences on visuomotor functioning.

Key words: eye movements; heartbeat; microsaccades

\section{Significance Statement}

In the present study, we show that microsaccades are coupled to heartbeat. Moreover, we revealed a strong modulation of slow eye movements around the R peak in the ECG. These results suggest that heartbeat as a basic physiological signal is related to statistical modulations of fixational eye movements, in particular, the generation of microsaccades. Therefore, our findings add a new perspective on the principles underlying the generation of fixational eye movements. Importantly, our study highlights the need to record eye movements when studying the influence of heartbeat in neuroscience to avoid misinterpretation of eye-movementrelated artifacts as heart-evoked modulations of neural processing.

\section{Introduction}

During visual fixation, the eyes continue to move on a smaller scale, producing fixational eye movements (FEMs) categorized as physiological drift (slow eye movements) and microsaccades that are rapid, small-amplitude movements with a mean rate of $1-2 / \mathrm{s}$ (Steinman et al., 1973). Research on FEM strongly focuses on their underlying generating mechanisms and their possible function for visual processing (for reviews, see Rolfs, 2009; Hafed, 2011; Martinez-Conde et al., 2013).

Recent findings have established a fundamental role of both microsaccades and slow eye movements for vision, thus adding to the existing view that microsaccades and slow eye movements

Received June 9, 2015; revised Dec. 10, 2015; accepted Dec. 18, 2015.

Author contributions: S.O., C.W., R.K., O.P., and R.E. designed research; S.O. and C.W. performed research; S.O. and R.E. analyzed data; S.O., R.K., O.P., and R.E. wrote the paper.

This work was supported by a the Deutsche Forschungsgemeinschaft Research Unit 868 to (Grant EN 471/3-2 to R.E. and Grant P0 1011/1-1 to 0.P.).

The authors declare no competing financial interests.

Correspondence should be addressed to Dr. Sven Ohl, Humboldt Universität zu Berlin, Bernstein Center for

Computational Neuroscience, Arbeitsgruppe Martin Rolfs, Philippstr. 13, Haus 6, 10115 Berlin, Germany. E-mail: sven.ohl@bccn-berlin.de.

DOI:10.1523/JNEUROSCI.2211-15.2016

Copyright $\odot 2016$ the authors $\quad 0270-6474 / 16 / 361237-05 \$ 15.00 / 0$ prevent perceptual fading from happening (McCamy et al., 2014a). Moreover, microsaccades effectively counteract perceptual fading (Ditchburn et al., 1959; Martinez-Conde et al., 2006; McCamy et al., 2012); however, fading does not causally trigger microsaccades (Poletti and Rucci, 2010). There is, however, a higher probability for the triggering of a microsaccade if retinal image motion is slower than average (Engbert and Mergenthaler, 2006; Engbert et al., 2011).

For the current study, we highlight two important properties of microsaccades. First, microsaccades have been observed to support vision in a high-acuity task (Ko et al., 2010). When participants were asked to thread a needle, microsaccades shifted the center of gaze between the needle and the thread back and forth. It seems that microsaccades fulfill the same purpose as large saccades, precisely shifting gaze to the most relevant locations to achieve the goal of a task (Kowler and Collewijn, 2010). This claim is further supported by the finding that visual sensitivity is not homogenous across the fovea and microsaccades bring the stimulus onto the preferred retinal locus of fixation (Poletti et al., 2013). Moreover, an excess of microsaccades is observed during inspection of informative scene regions, suggesting a role of microsaccades in the sampling of visual information (McCamy et al., 2014b). 
Second, fixational drift has been shown to improve discrimination of high-spatial-frequency stimuli (Rucci et al., 2007). The small, ongoing movements of the eye during fixation do not represent a disturbing signal, but rather help the visual system to process fine spatial details. The beneficial role of fixational drift is thought to unfold already during retinal processing by redistributing the temporal frequency power into a range of increased retinal sensitivity (Kuang et al., 2012) and by decorrelating highly redundant input from natural scenes (Rucci and Victor, 2015). Therefore, the view has emerged that fixational drift is adapted to an image to transform visual signals into an optimized spatiotemporal code for further visual processing (Kagan, 2012).

In the present study, we add a new perspective on FEM: the role of heartbeat on its generation. Whereas human observers fixated a centrally presented point, we simultaneously recorded their eye positions and ECG. As a result, we observed an unexpected and strong coupling of microsaccade onsets and heartbeat, demonstrating a physiological modulation of the probability for microsaccade generation.

\section{Materials and Methods}

Participants. Eighteen observers ranging in age from 18 to 29 years (mean $=22.1$ years, 7 female) with normal or corrected-to-normal vision participated in the present study. The study followed the ethical standards from the Declaration of Helsinki and observers' informed consent was obtained before the experiment. Observers received study credit or were paid 7 Euros for participation of the $1 \mathrm{~h}$ experiment.

Task and procedure. Before trial onset, a white fixation cross was displayed (width, height of $0.45^{\circ}$ ) and a fixation routine controlled whether observers fixated the centrally presented cross. After successful fixation control, the cross was removed at trial onset and replaced by a white tiny fixation point (diameter of $0.1^{\circ}$ ) in the center of the screen on a black background. Subjects were asked to keep fixating this point for the complete trial duration of $20 \mathrm{~s}$. Observers were instructed to avoid blinking because blinks were automatically detected, leading to trial abort. Such trials were repeated at the end of the experiment such that each observer completed a total of 30 trials.

Between trials, pictures with natural scenes were shown for $5 \mathrm{~s}$, allowing observers to move their eyes and have a short break. By button press, observers could initialize the next trial. Standard nine-point calibration was conducted to align eye and screen coordinate systems. If necessary, calibration and validation procedures were run before every trial. Otherwise, an automatic routine caused calibration and validation procedures every five trials.

Apparatus. Visual stimuli were presented on a 22 -inch FT/LCD monitor with a $60 \mathrm{~Hz}$ refresh rate and a resolution of $1680 \times 1050$ pixel. Observers were seated at a distance of $50 \mathrm{~cm}$ from the screen with their head stabilized on a chin rest. Stimuli were generated and displayed using Python and the Vision Egg toolbox (Straw, 2008). Eye positions of both eyes were recorded with a sampling frequency of 500 $\mathrm{Hz}$ using the Eyelink-II system (SR Research). ECG was recorded at a sampling frequency of $1000 \mathrm{~Hz}$ using the Biopac MP36 system, placing electrodes on the left and the right arm and left leg according to Einthoven.

Data preprocessing. Microsaccades were detected using a velocitybased algorithm (Engbert and Kliegl, 2003; Engbert and Mergenthaler, 2006). Raw eye positions were transformed into velocity space and segments that passed a velocity threshold of 5 median-based SD for a minimum duration of $8 \mathrm{~ms}$ were classified as microsaccades. Only binocular microsaccades were included for further analyses. Moreover, we excluded all eye movements larger than a $1.5^{\circ}$ of visual angle.

We identified the first $\mathrm{R}$ peaks in the ECG signal by computation of maxima in the ECG trace. The first two maxima that exceeded the $98^{\text {th }}$ percentile of voltage values in the ECG allowed reliable detection of the first $\mathrm{R}$ peaks as controlled by visual inspection. In a second step, we searched for R peaks in a new time window. To this end, we moved the window stepwise by an adjustable duration that was defined by the length of the interval between the last two $\mathrm{R}$ peaks. We visually inspected detection performance of $\mathrm{R}$ peaks yielded by the algorithm and applied small parameter adjustments whenever necessary.

During preprocessing, we had to exclude three observers from further data analysis. For two observers, the ECG recordings were very noisy and allowed identification of $\mathrm{R}$ peaks in $<65 \%$ of the trials. For one participant, only eye positions were recorded, thus missing the ECG.

Data analysis. For each microsaccade, we determined the onset of the previous and following R peak from observers' ECG. Based on this information, we computed the relative phase of microsaccade onset within the interval between two $\mathrm{R}$ peaks, assigning values between 0 and $2 \pi$ (Schäfer et al., 1998; Pikovsky et al., 2001). For each individual observer, we computed circular densities (bandwidth $=10$ for density computation) and compared these circular densities with the null hypothesis of a uniform circular density. Statistical significance was determined using the following bootstrapping procedure. A single bootstrap sample consisted of 15 randomly drawn (with replacement) participants from the original pool of 15 participants. For each participant in this bootstrap sample, we computed the circular density of microsaccades in a heart phase and computed the mean circular density across the 15 participants in the bootstrap sample. We repeated this procedure 1000 times and determined median and the $95 \%$ confidence interval based on these 1000 bootstrap samples. We consider segments of the heart phase as significant when the circular density of a uniform distribution is outside of the $95 \%$ confidence interval determined by the described bootstrapping procedure.

Individual confidence intervals for each participant were computed by resampling $n$ relative microsaccade onsets from the observed distribution of relative microsaccades onsets for that given participant (consisting of $n$ values). We repeated this resampling routine 1000 times, which allowed us to compute $95 \%$ confidence intervals for each individual observer.

Ocular drift (retinal image slip) was quantified using a box-count analysis (Engbert and Mergenthaler, 2006). To do so, the eye trajectory for a given time window is extracted and the number of squared boxes (box width $=0.01^{\circ}$ ) necessary to cover the eye trajectory is determined. The necessary number of boxes was determined for the eye trajectory in a time window of $50 \mathrm{~ms}$. Moving this window by steps of $50 \mathrm{~ms}$ allowed us to compute the time course of retinal image slip locked to the R peak in the ECG. The time course ranged from 1000 $\mathrm{ms}$ before the R peak to $1000 \mathrm{~ms}$ after the R peak (in steps of $50 \mathrm{~ms}$ ). To remove potential confounding influences induced by microsaccades, segments in the eye trajectory, including microsaccades, were excluded for the analysis. More specifically, when microsaccades occurred within $800 \mathrm{~ms}$ before the R peak, parts from the eye trajectory were excluded from $800 \mathrm{~ms}$ before the R peak to microsaccade onset plus $50 \mathrm{~ms}$ (locked to $\mathrm{R}$ peak). Accordingly, when microsaccades occurred within $800 \mathrm{~ms}$ after the R peak, parts of the eye trajectory were excluded starting from microsaccade onset minus $50 \mathrm{~ms}$. The box count analysis was run separately for each eye and then averaged across both eyes.

Similar to the box count analysis, we determined the pupil size in a range from $1000 \mathrm{~ms}$ before the R peak to $1000 \mathrm{~ms}$ after the R peak. For each individual observer, we computed $z$-transformed pupil sizes and computed the same analysis as for the box count to determine the time course around the $\mathrm{R}$ peak.

\section{Results}

In our study, observers were asked to maintain fixation on a centrally presented fixation spot for the duration of $20 \mathrm{~s}$. Microsaccades were detected in eye traces obtained from $15 \mathrm{ob}-$ servers, each running 30 trials. The observed mean rate of 1.04 microsaccades/s (range across observers: $0.38-2.39$ microsaccades/s) is in good agreement with earlier studies on microsaccade rate (Engbert and Kliegl, 2003). For a total of 9230 microsaccades, we determined the onset's relative phase to the preceding and following R peak of the QRS complex in the 


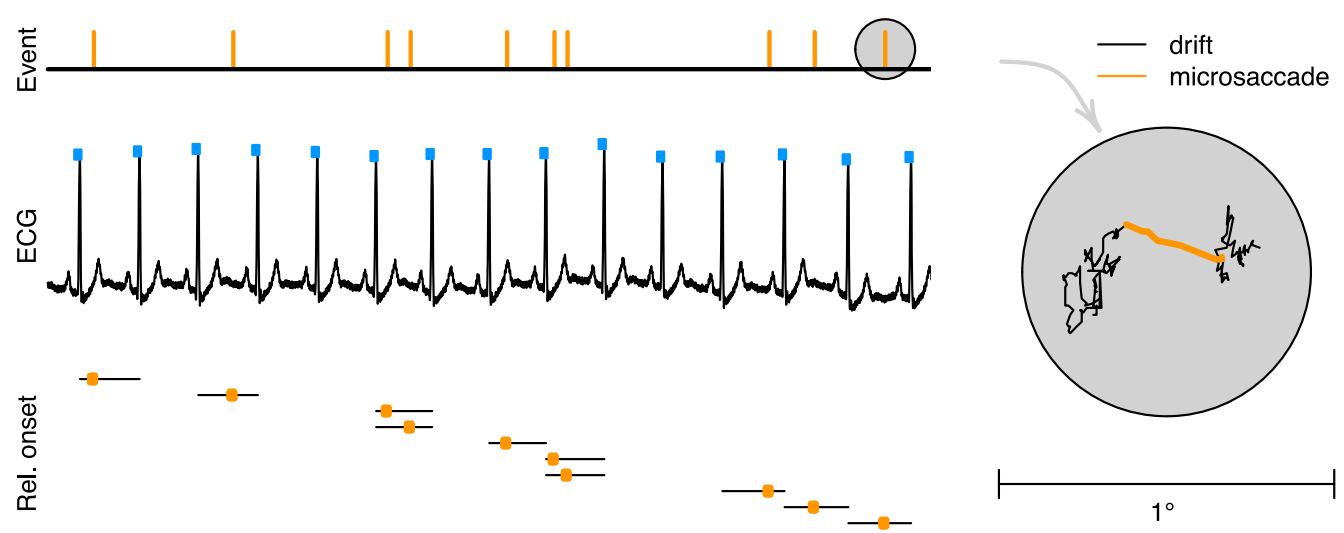

Figure 1. Coupling of FEM and heartbeat (R peaks). Microsaccades (orange) and R peaks in the ECG (blue) are detected simultaneously. Microsaccade onset is determined relative to the preceding and following $\mathrm{R}$ peak.

a

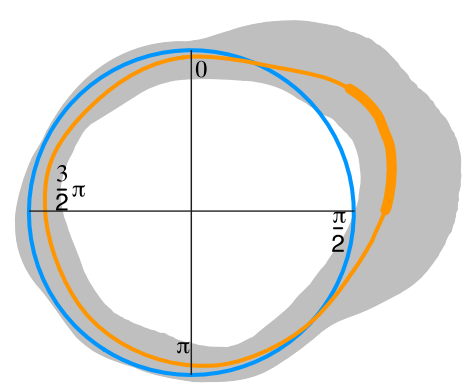

b

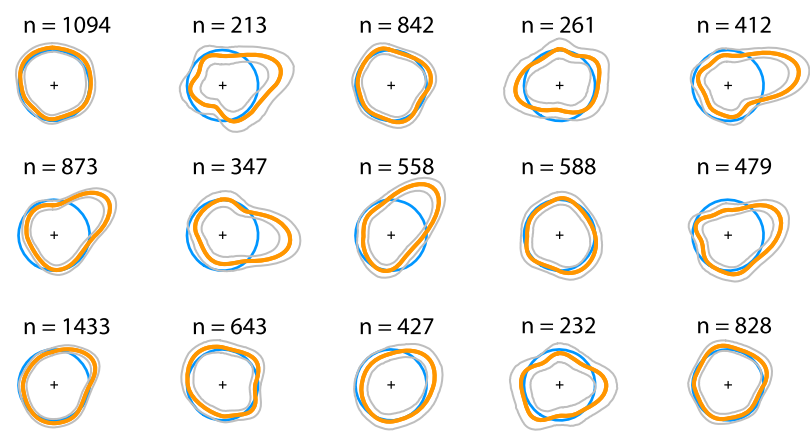

Figure 2. $\quad \boldsymbol{a}$, Deviation from null hypothesis of uniformly distributed microsaccades in the interval between two R peaks. Shown is a comparison of circular density of R-peak-locked microsaccades (orange; $95 \%$ confidence interval in gray) and circular density of a uniform distribution (blue). $\boldsymbol{b}$, Circular densities of relative microsaccade onsets for each individual observer (orange), along with the $95 \%$ confidence interval (gray). Computation is based on resampling from the original pool of microsaccades of a given participant. In addition, the number of microsaccades for each observer is presented on top of the circular densities.

ECG signal and assigned corresponding values of relative phase ranging between 0 and $2 \pi$ (Fig. 1 ).

We tested the coupling of microsaccades and heartbeat by analyzing the distribution of relative microsaccade onsets between two R peaks. We computed circular densities of relative microsaccade onsets and compared those to a circular uniform distribution. A significant coupling of microsaccades and heartbeat is indicated when the circular density of relative microsaccade onsets differs significantly from a uniform distribution (Fig. 2). Indeed, significantly more microsaccades were generated during the early phase in the cardiac cycle (i.e, from 0.29 to $0.50 \pi)$. Therefore, by coregistration of eye position and heartbeat signals, we found statistical evidence for a significant coupling of microsaccades and heartbeat.

Recent research supported the idea of an interaction between drift and microsaccades. Image slip (expressed as box count measure indicating the area covered by the drift movement) decreased before microsaccade onset (Engbert and Mergenthaler, 2006) and increased after microsaccade offset (Chen and Hafed, 2013). We investigated whether retinal image slip is correlated with heartbeat and observed a strong rhythmic coupling with heartbeat (Fig. 3a). Image slip decreases starting $500 \mathrm{~ms}$ before $\mathrm{R}$ peak, reaching a minimum plateau around the $\mathrm{R}$ peak and increasing later on. Therefore, in addition to a coupling with microsaccades, heartbeat also correlates with physiological drift.

We also computed the temporal evolution of pupil size locked to R peak (Fig. $3 b$ ) and observed some covariation of pupil size and heartbeat. However, the analysis of pupil size around the $\mathrm{R}$ peak indicates strong interindividual differences of the coupling between pupil size variation and heartbeat, whereas the temporal modulation of retinal image motion around the $\mathrm{R}$ peaks is very consistent across participants. We derived effect sizes for both image slip and pupil size around the R peak by computing the ratio of maximum amplitude of the temporal evolution (in a comparable time window) and the maximum standard error in that time window. The computed effect sizes of 13.21 for image slip (amplitude of 3.17 in the time window -500 to $0 \mathrm{~ms}$ divided by the maximum SE of 0.24 in that time window) and 2.35 for pupil size (amplitude of 0.04 in the time window -350 to $0 \mathrm{~ms}$ divided by the maximum SE of 0.017 in that time window) further show that the temporal evolution of image slip around $\mathrm{R}$ peak is $>5$ times stronger than for pupil size.

\section{Discussion}

The results of the present study suggest that one's own heartbeats constitute a physiological signal that is related to the generation of FEM. This finding demonstrates a coupling of heartbeat and the oculomotor system and therefore adds to the growing literature of bodily influences on perception (Gray et al., 2007; Park et al., 2014).

Several hypotheses should be addressed in future research to understand the coupling of heartbeat and FEM. First, multiple heartbeat-evoked potentials affect neural processing (Schandry and Montoya, 1996; Pollatos et al., 2007; Kern et al., 2013), with 

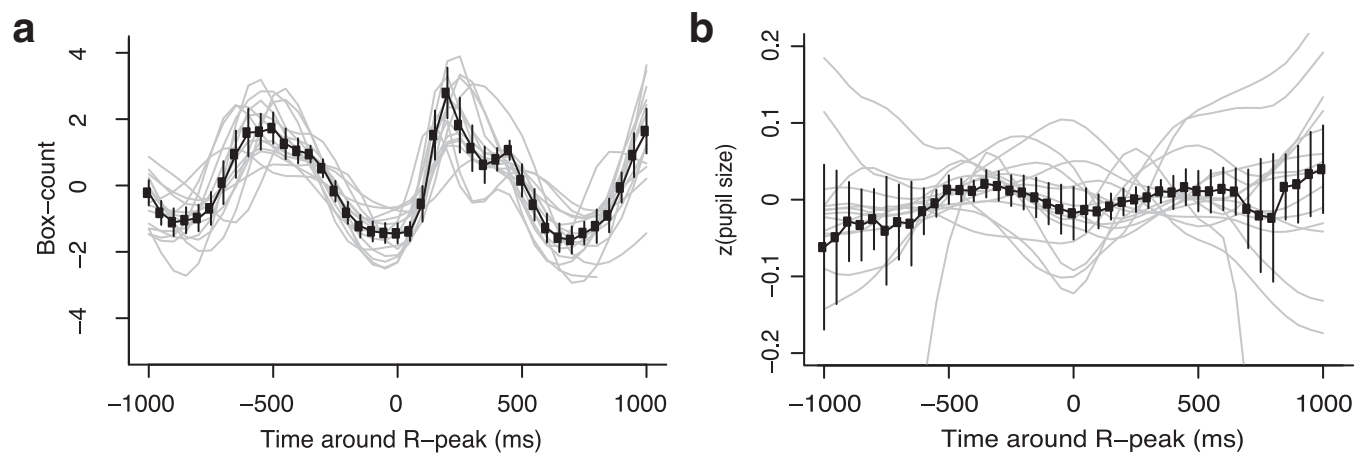

Figure 3. $a$, Box count analysis showing the number of boxes (width $=0.01^{\circ}$ ) necessary to cover the eye trajectory in a time window of $50 \mathrm{~ms}$. Mean ( $\pm 95 \%$ confidence interval) centered box count is shown in black and the smoothed time course for each observer in gray (spline, $\mathrm{df}=15$ ). $\boldsymbol{b}$, Time course of pupil size around the R peak. Mean ( $\pm 95 \%$ confidence interval) $z$-transformed pupil size is shown in black and smoothed time course for each observer in gray (spline, $\mathrm{df}=15$ ).

marked consequences for sensory processing contingent on the presentation of a stimulus during systole versus diastole (Rau and Elbert, 2001). Such heartbeat-related signals in the visual system could modulate the perception of fine detail via stochastic resonance (Simonotto et al., 1997) and subsequently triggered microsaccades.

Alternatively, heartbeat-evoked potentials in oculomotor regions (e.g., superior colliculus; Hafed et al., 2009) could affect the generation of microsaccades directly. Recently proposed computational models of microsaccade generation demonstrate how neural dynamics in the superior colliculus could trigger microsaccades. From the perspective of the neural equilibrium distribution (Goffart et al., 2012), heartbeat-evoked neural responses induce spontaneous fluctuations in the superior colliculus oculomotor map. This in turn would generate a microsaccade if the activity distribution's center of mass deviated significantly from the center of the map. From the perspective of neural dynamics (Engbert et al., 2011), heartbeat drives slow fixational eye movements by oscillatory changes of the movement's potential that constrains the current focus of visual selective attention (Hafed and Clark, 2002; Engbert and Kliegl, 2003). Such a mechanism has been shown to modulate microsaccade rates (Engbert, 2012) and might be implemented in the superior colliculus. Indeed, the coupling of microsaccade statistics and visual attention is disrupted after suppression of activity in the superior colliculus (Hafed et al., 2013).

Second, the observed influence of heartbeat on FEM could be the result of direct influences on eye muscle activity. In this case, noisy variations of heart activity produce variations of eye's muscle receptor activation (Fallon et al., 2004), which modulate slow fixational eye movements. Therefore, modulations of slow FEM generate temporal variations in microsaccades (Engbert and Mergenthaler, 2006). Third, both modulations of heartbeat and FEM could be triggered by an unobserved common cause (e.g., attention, arousal, or orienting response; Sokolov, 1960), thus raising the need to further scrutinize the causal relationship in future studies.

Whether one of our hypotheses on the neural sources can be falsified or not, the current findings have important general implications for experiments in the field of neuroscience. Because microsaccades evoke gamma-band activity in the EEG (YuvalGreenberg et al., 2008; Dimigen et al., 2009), our results on the coupling of microsaccades and heartbeat indicate the need for recording FEM and ECG to reduce systematic influences of ocular artifacts on electrophysiological signals that are interpreted as brain activity. This is particularly important as more studies be- gin to focus on the influence of heartbeat in visual neuroscience (Park et al., 2014; Winston and Rees, 2014).

\section{References}

Chen CY, Hafed ZM (2013) Postmicrosaccadic enhancement of slow eye movements. J Neurosci 33:5375-5386.

Dimigen O, Valsecchi M, Sommer W, Kliegl R (2009) Human microsaccade-related visual brain responses. J Neurosci 29:12321-12331.

Ditchburn RW, Fender DH, Mayne S (1959) Vision with controlled movements of the retinal image. J Physiol 145:98-107.

Engbert R (2012) Computational modeling of collicular integration of perceptual responses and attention in microsaccades. J Neurosci 32:8035-8039.

Engbert R, Kliegl R (2003) Microsaccades uncover the orientation of covert attention. Vis Res 43:1035-1045.

Engbert R, Mergenthaler K (2006) Microsaccades are triggered by low retinal image slip. Proc Natl Acad Sci USA 103:7192-7197.

Engbert R, Mergenthaler K, Sinn P, Pikovsky A (2011) An integrated model of fixational eye movements and microsaccades. Proc Natl Acad Sci USA 108:E765-E770.

Fallon JB, Carr RW, Morgan DL (2004) Stochastic resonance in muscle receptors. J Neurophysiol 91:2429-2436.

Goffart L, Hafed ZM, Krauzlis RJ (2012) Visual fixation as equilibrium: Evidence from superior colliculus inactivation. J Neurosci 32:1062710636.

Gray MA, Taggart P, Sutton PM, Groves D, Holdright DR, Bradbury D, Brull D, Critchley HD (2007) A cortical potential reflecting cardiac function. Proc Natl Acad Sci USA 104:6818-6823.

Hafed ZM (2011) Mechanisms for generating and compensating for the smallest possible saccades. Eur J Neurosci 33:2101-2113.

Hafed ZM, Clark JJ (2002) Microsaccades as an overt measure of covert attention shifts. Vis Res 42:2533-2545.

Hafed ZM, Goffart L, Krauzlis RJ (2009) A neural mechanism for microsaccade generation in the primate superior colliculus. Science 323:940-943.

Hafed ZM, Lovejoy LP, Krauzlis RJ (2013) Superior colliculus inactivation alters the relationship between covert visual attention and microsaccades. Eur J Neurosci 37:1169-1181.

Kagan I (2012) Active vision: Fixational eye movements help seeing space in time. Curr Biol 22:R186-R188.

Kern M, Aertsen A, Schulze-Bonhage A, Ball T (2013) Heart cycle-related effects on event-related potentials, spectral power changes, and connectivity patterns in the human ECoG. Neuroimage 81:178-190.

Ko H-K, Poletti M, Rucci M (2010) Microsaccades precisely relocate gaze in a high visual acuity task. Nat Neurosci 13:1549-1553.

Kowler E, Collewijn H (2010) The eye on the needle. Nat Neurosci 13: 1443-1444.

Kuang X, Poletti M, Victor JD, Rucci M (2012) Temporal encoding of spatial information during active visual fixation. Curr Biol 22:510-514.

Martinez-Conde S, Macknik SL, Troncoso XG, Dyar TA (2006) Microsaccades counteract visual fading during fixation. Neuron 49:297-305.

Martinez-Conde S, Otero-Millan J, Macknik SL (2013) The impact of microsaccades on vision: towards a unified theory of saccadic function. Nat Rev Neurosci 14:83-96. 
McCamy MB, Otero-Millan J, Macknik SL, Yang Y, Troncoso XG, Baer SM, Crook SM, Martinez-Conde S (2012) Microsaccadic efficacy and contribution to foveal and peripheral vision. J Neurosci 32:9194-9204.

McCamy MB, Macknik SL, Martinez-Conde S (2014a) Different fixational eye movements mediate the prevention and the reversal of visual fading. J Physiol 592:4381-4394.

McCamy MB, Otero-Millan J, Di Stasi LL, Macknik SL, Martinez-Conde S (2014b) Highly informative natural scene regions increase microsaccade production during visual scanning. J Neurosci 34:2956-2966.

Park H-D, Correia S, Ducorps A, Tallon-Baudry C (2014) Spontaneous fluctuations in neural responses to heartbeats predict visual detection. Nat Neurosci 17:612-618.

Pikovsky A, Rosenblum M, Kurths J (2001) Synchronization: a universal concept in nonlinear sciences. Cambridge: Cambridge University.

Poletti M, Rucci M (2010) Eye movements under various conditions of image fading. J Vis 10:1-18.

Poletti M, Listorti C, Rucci M (2013) Microscopic eye movements compensate for nonhomogeneous vision within the fovea. Curr Biol 23:1691-1695.

Pollatos O, Schandry R, Auer DP, Kaufmann C (2007) Brain structures mediating cardiovascular arousal and interoceptive awareness. Brain Res 1141:178-187.

Rau H, Elbert T (2001) Psychophysiology of arterial baroreceptors and the etiology of hypertension. Biol Psychol 57:179-201.
Rolfs M (2009) Microsaccades: Small steps on a long way. Vis Res 49:2415-2441.

Rucci M, Victor JD (2015) The unsteady eye: an information-processing stage, not a bug. Trends Neurosci 38:195-206.

Rucci M, Iovin R, Poletti M, Santini F (2007) Miniature eye movements enhance fine spatial detail. Nature 447:852-855.

Schäfer C, Rosenblum MG, Kurths J, Abel HH (1998) Heartbeat synchronized with ventilation. Nature 392:239-240.

Schandry R, Montoya P (1996) Event-related brain potentials and the processing of cardiac activity. Biol Psychol 42:75-85.

Simonotto E, Riani M, Seife C, Roberts M, Twitty J, Moss F (1997) Visual perception of stochastic resonance. Phys Rev Lett 78:1186-1189.

Sokolov EN (1960) Neuronal models and the orienting reflex. In: The central nervous system and behavior (Brazier MAB, ed), pp 187-276. New York: JosiahMacy, Jr. Foundation.

Steinman RM, Haddad GM, Skavenski AA, Wyman D (1973) Miniature eye movement. Science 181:810-819.

Straw AD (2008) Vision Egg: an open-source library for realtime visual stimulus generation. Front Neuroinform 2:1-10.

Winston JS, Rees G (2014) Following your heart. Nat Neurosci 17:482-483.

Yuval-Greenberg S, Tomer O, Keren AS, Nelken I, Deouell LY (2008) Transient induced gamma-band response in EEG as a manifestation of miniature saccades. Neuron 58:429-441. 